\title{
Improving ATV's stability by means of a gyroscopic mechanism
}

\author{
Iliyana Minkovska ${ }^{1, *}$, Georgi Kadikyanov ${ }^{1}$, Rosen Ivanov ${ }^{1}$, and Rusi Rusev ${ }^{1}$ \\ ${ }^{1}$ University of Ruse, Department of Engines and Vehicles, Rousse, Bulgaria
}

\begin{abstract}
This paper presents research carried out to improve ATV stability by means of a built-in gyroscopic mechanism. For this purpose, a mathematical model was used. The negative gyroscopic effect of wheel rotation on ATV stability is considered. The theoretical results for ATV motion and the impact of the built-in gyroscopic mechanism on its stability under various conditions are presented.
\end{abstract}

\section{Introduction}

The growing interest in ATV driving increases the risks of accidents. Most frequently, the reason why they occur is stability loss when ATV moves in curves [1,2]. Loss of stability has two aspects: lateral sliding and lateral overturn. Depending on the different performance (grip coefficient, radius of curve, center of gravity coordinates in ATV riding by different riders, etc.) when ATV moves in curves, one of the above possibilities to lose stability may arise. It is essential which one will come first. The stability loss for lateral overturn presents a greater risk to the life and health of the driver (Fig. 1).

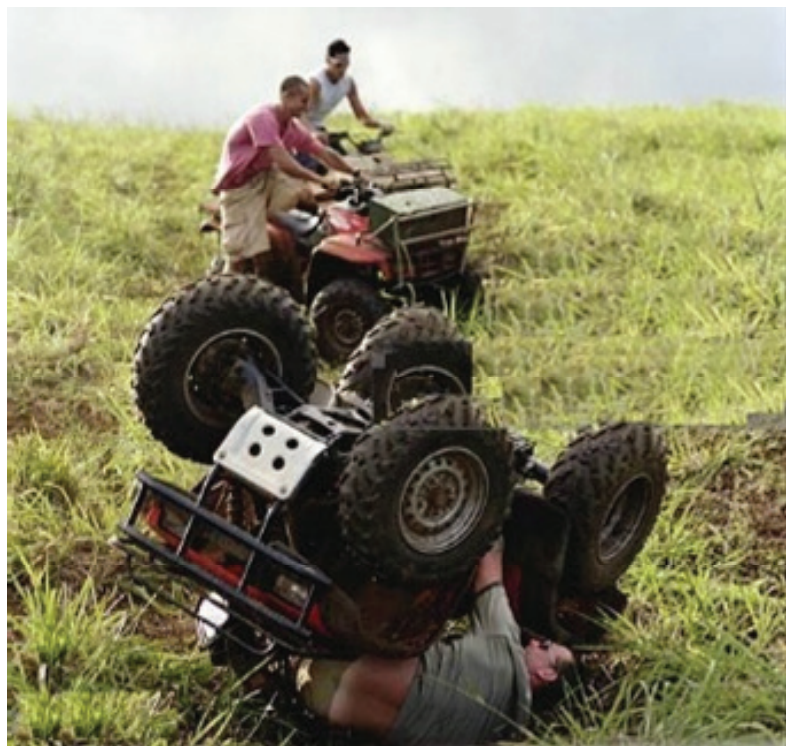

Fig. 1. Stability loss at lateral overturn with a risk for the life and health of the driver.

To improve the stability at overturn, a built-in gyroscopic mechanism in the ATV structure is useful [3]. It is a metal solid disk with high angular rotation speed. The gyroscopic mechanism axis is parallel to the transverse horizontal of vehicle. In this way the gyroscopic moment is used when ATV makes turns [4].
This feature is may be negative to the ATV controllability. On the other hand, the appropriate axis allocation and proper rotation direction may be used to improve the stability of movement in curves.

The main difficulty in using such devices comes from the danger to break the rotor because of the forces occurring at the high angular rotation speed. To prevent the consequences of device breaking (for monolithic rotor), it should be placed in a hard-to-break container.

The aim of this study is to determine the possibility to improve the stability of ATV in curve movement by means of a built-in gyroscopic mechanism.

\section{Methodology of mathematical calculations}

Due to the kinematics of wheel vehicles, a gyroscopic moment arises when wheels rotate, which negatively influences controllability and stability. To calculate the gyroscopic moment of the four wheels equation (1) is used:

$$
M_{G k}=4 J_{k} \omega_{k} \Omega, \mathrm{Nm},
$$

where

$J_{k}, \mathrm{~kg} \cdot \mathrm{m}^{2}$ is the wheels mass moment of inertia;

$\omega_{k}, \mathrm{rad} / \mathrm{s}$ - angular rotation speed of wheels;

$\Omega, \mathrm{rad} / \mathrm{s}$ - angular rotation speed of ATV around the center of curve.

The wheels mass moment of inertia is determined by the standard methods through an experimental device shown in Fig. 2.

According to [5] under the different operation modes the critical velocity, whichever is lower, against lateral sliding and lateral overturn is taken for calculating $\Omega, \mathrm{rad} / \mathrm{s}$ - angular rotation speed of ATV around the center of curve.

When determining the critical velocities in [5] a two-mass model is used for selecting the lower of velocities on the front or rear drive axles. Only cases for which the grip coefficient is 0,75 are considered.

\footnotetext{
Corresponding author: iminkovska@,uni-ruse.bg
} 


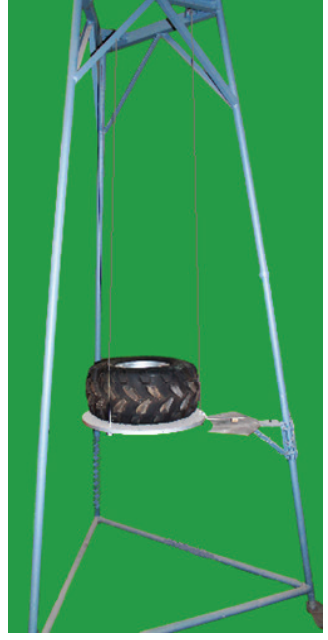

Fig. 2. Experimental device to determine the mass moment of inertia of the wheels.

In cases where the riders who are with higher BMI (body mass index [6]) the critical velocities against overturn are lower than the critical velocities against lateral sliding.

Figure 3 shows a schematic diagram of the forces acting on the ATV with no built-in gyroscopic mechanism when it moves in a curve.

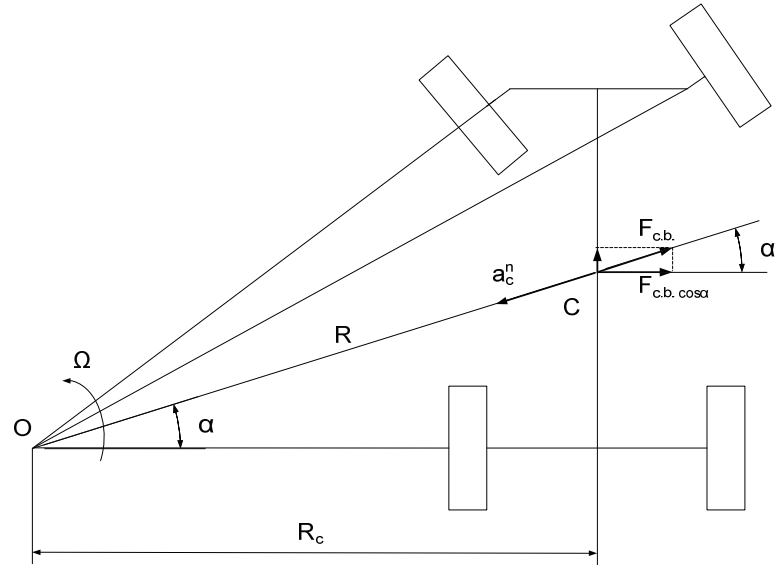

Fig. 3. Schematic diagram of the forces acting on the ATV with no built-in gyroscopic mechanism when it moves in a curve.

The angular rotation speed of ATV around the center of curve according to Fig. 3 is

$$
\Omega=\frac{V_{a}}{R} \mathrm{rad} / \mathrm{s} .
$$

In theoretical calculations of the gyroscopic mechanism for the purposes of design and layout, the following values are assumed: disk diameter $d=300 \mathrm{~mm}$ and disk width $b=30 \mathrm{~mm}$. For steel disk with permissible tension of $\sigma_{T}=400 \mathrm{MPa}$, density $\rho=7850 \mathrm{~kg} / \mathrm{m}^{3}$, radius $r=0,15 \mathrm{~m}$, the maximum permissible rotation speed will be

$$
\omega=\sqrt{\frac{\sigma_{T}}{\rho r^{2}}}, \mathrm{rad} / \mathrm{s}
$$

$$
n=\frac{30 \omega}{\pi}, \min ^{-1} .
$$

The gyroscopic mechanism is positioned in the middle of the rear part of vehicle. Its minor influence on the change of center of gravity coordinates of ATV is ignored.

The gyroscopic stabilizing moment will be

$$
M_{G s}=J_{d} \omega_{d} \Omega, \mathrm{Nm},
$$

where

$J_{d}, \mathrm{~kg} \cdot \mathrm{m}^{2}$ is the disk mass moment of inertia; $\omega_{d}, \mathrm{rad} / \mathrm{s}-$ disk angular rotation speed.

The centripetal acceleration is

$$
a_{c}^{n}=R \Omega^{2}, \mathrm{~m} / \mathrm{s}^{2} .
$$

The centrifugal force corresponding to the critical velocities $V_{a}$ from [5] without embedded gyroscope is

$$
F_{c . b .}=a_{c}^{n} m_{A}, \mathrm{~N},
$$

where

$m_{A}, \mathrm{~kg}$ is the total mass: of ATV mass and rider.

Then, under these conditions for the stabilizing moment the following is obtained

$$
M_{s}=F_{c . b .} h_{A} \cos \alpha, \mathrm{Nm},
$$

where

$h_{A}, \mathrm{~m}$ is the vertical coordinate of the center of gravity; $\alpha,{ }^{\circ}$ - the angle between the centrifugal force directrix and the axis of rear drive axle.

The total stabilizing moment regarding the gyroscopic effects is

$$
M_{o}=M_{G s}+M_{s}-M_{G k}, \mathrm{Nm} .
$$
is

The centrifugal force corresponding to this moment

$$
F_{c . b . o}=\frac{M_{o}}{h_{A} \cos \alpha}, \mathrm{Nm} \text {. }
$$

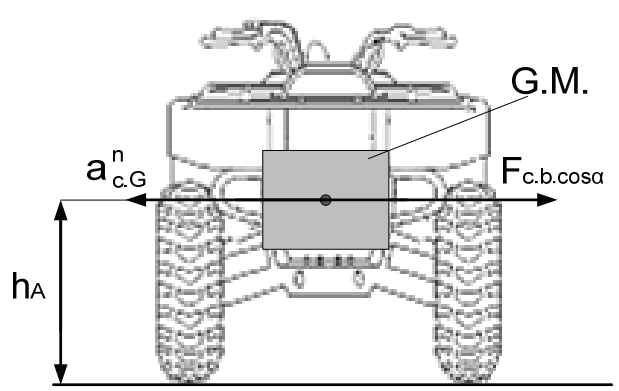

Fig. 4. Schematic diagram of the forces acting on the ATV with a built-in gyroscopic mechanism in transverse plane when it moves in a curve.

According to Fig. 4 the centripetal acceleration under the action of the total stabilizing moment is 


$$
a_{c G}^{n}=\frac{F_{c . b . o}}{m_{A}}, \mathrm{~m} / \mathrm{s}^{2} .
$$

Angular rotation speed of ATV around the center of the curve with centripetal acceleration according to (6) and (11) is

$$
\Omega_{o}=\sqrt{\frac{a_{c G}^{n}}{R}}, \mathrm{rad} / \mathrm{s}
$$

Then the critical velocity against overturn taking into account the gyroscopic moments: the effect of wheels rotation and the built-in gyroscopic mechanism is

$$
V_{A, G}=\Omega_{o} R, \mathrm{~m} / \mathrm{s} .
$$

The critical velocities obtained from equation (13) are compared to the critical velocities obtained in [5] for 0,75 value of the grip coefficient.

\section{Results of the study}

The critical velocities against lateral sliding and lateral overturn when taking into account the effect of the gyroscopic mechanism built in ATV for riders with different body mass index (BMI) is shown in Figures 5 to 9 .

It can be seen from Figures 5 to 9 that the critical velocities against lateral sliding for radiuses of curves bigger than $10 \mathrm{~m}$ and $15 \mathrm{~m}$ do not depend on the BMI of different riders. This is so because these velocities are not affected by the mass of the vehicle or rider. For smaller $3 \mathrm{~m}$ and $6 \mathrm{~m}$ radiuses of curves, the critical velocities against lateral sliding differ for some of the riders as the loss of stability occurs at either rear or front axles.

The critical velocities against lateral overturn for riders with higher BMI (more than 30 for any radius of curves, and more than 25 for radius of curve $3 \mathrm{~m}$ ) are lower than those against lateral sliding. This is very dangerous as the loss of stability with lateral overturn occurs all of the sudden. Particularly for riders with higher BMI it occurs because of the higher vertical coordinate of the center of gravity.

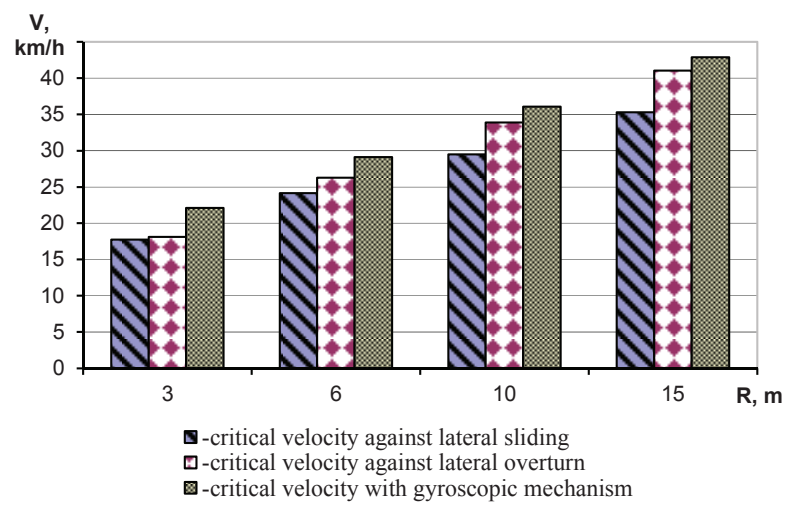

Fig. 5. Critical ATV driving velocities with a rider of BMI 21.

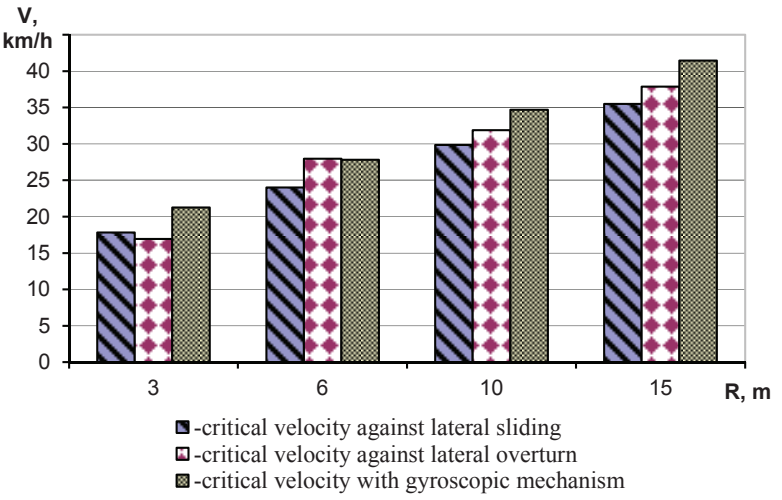

Fig. 6. Critical ATV driving velocities with a rider of BMI 25.

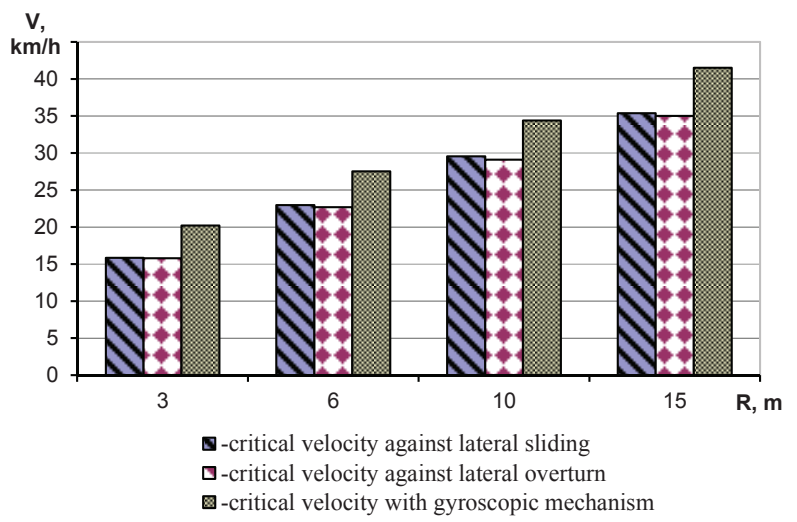

Fig. 7. Critical ATV driving velocities with a rider of BMI 30.

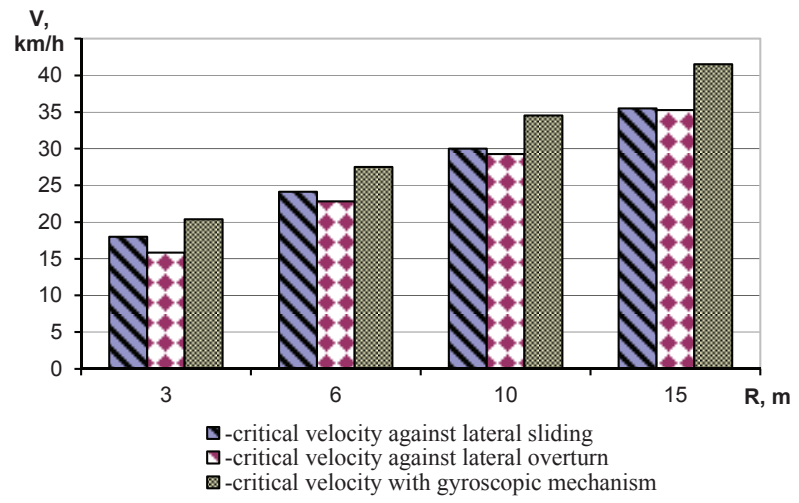

Fig. 8. Critical ATV driving velocities with a rider of BMI 31.

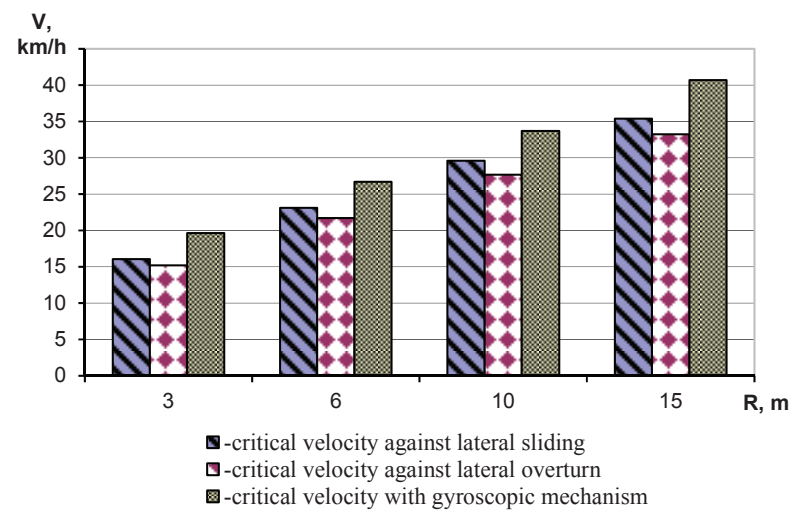

Fig. 9. Critical ATV driving velocities with a rider of BMI 40. 
It can be seen from the graphics that the critical velocities against overturn increase with a buildt-in gyroscopic mechanism in ATV which prevents its occurrence. In such cases the critical velocity against lateral sliding remains lower. Then, for all riders, at first the lateral sliding will occur. This phenomenon is not so risky for the riders' health and life.

\section{Conclusions}

1. For riders with lower BMI of up to 25 , the gyroscopic mechanism has no influence on the ATV stability. In such cases lateral sliding occurs before reaching lateral overturn velocities.

2. For riders with higher BMI, embedding a gyroscopic mechanism increases the critical velocity against lateral overturn by up to $5 \%$ for a rider with BMI 40.

3. Increasing the critical velocity against lateral overturn by embedding a gyroscopic mechanism in the cases considered guarantees that the less dangerous phenomenon of lateral sliding will appear at first.

The study was supported by contract BG05M2OP001-2.0090011-C01 of University of Ruse "Angel Kanchev", "Support for the development of human resources for research and innovation at the University of Ruse "Angel Kanchev". The project is funded with support from the Operational Program "Science and Education for Smart Growth 2014 - 2020" financed by the European Social Fund of the European Union.

\section{References}

1. D. Blower, J. Woodrooffe, P. Green, A. Matteson, M. Shrank, Determination of events leading to sport utility vehicle rollovers. Transport. Res. Record 1908. 180-186, (2005).

2. S. Tanner, M. Aitken, J. Warnock, Analysis of All Terrain Vehicle Crash Mechanisms. Journal of undergraduate research bioengineering, 53-58 (2010)

3. R. Sharp, D. Limebeer, A motorcycle model for stability and control analysis, Multibody System Dynamics, 6(2), 123-142, (2001)

4. T. Sung, S. Han, Y. Han, J. Lee, N. Jeong, S. Hwang, $\mathrm{S}$. Choi, Designs and analyses of flywheel energy storage systems using high-Tc superconductor bearings. Cryogenics, 42, 357-362, (2002)

5. G. Kadikyanov, I. Minkovska, G. Staneva, A Study of the Influence of Some Exploitation Factors on the ATV Stability, Transport Problems, Katowice, 262269, (2017)

6. P. Deurenberg, M. Yap, W. VanStaveren, Body mass index and percent body fat: a meta analysis among different ethnic groups. Int. J. of Obesity, 1164-1171, (1998) 